\title{
Electron Energy Loss Spectroscopy of Vanadium Tetracyanoethylene
}

Amanda Trout $^{1}$, Seth Kurfman ${ }^{2}$, Michael Chilcote ${ }^{2}$, Ezekiel Johnston-Halperin ${ }^{2}$ and David McComb ${ }^{3}$

${ }^{1}$ The Ohio State University/Center for Electron Microscopy and Analysis, Dublin, Ohio, United States,

${ }^{2}$ The Ohio State University, Columbus, Ohio, United States, ${ }^{3}$ Center for Electron Microscopy and Analysis/ Department of Material Science and Engineering, The Ohio State University, Columbus, Ohio, United States

Vanadium tetracyanoethylene is an organic based magnet that has drawn much attention due to its high magnetic ordering temperature $(\sim 400 \mathrm{~K}) .^{1,2}$ Although the magnetic properties have been extensively studied since its discovery in $1991,{ }^{1}$ details of the magnetic mechanisms and electronic structure are still uncertain due to a lack of structural information. Characterization is challenging as many $\mathrm{V}(\mathrm{TCNE})_{\mathrm{X} \sim 2}$ films have high structural disorder or are amorphous, and the material is extremely air sensitive. Several structures have been proposed, and in general, the structure is believed to be a three dimensional network of $\mathrm{V}$ atoms in octahedral coordination with six $\mathrm{N}$ atoms from surrounding TCNE molecules. The valence state of the $\mathrm{V}$ is believed to be $2+$ though some have proposed a mixed valence state. ${ }^{1-5}$

Further characterization of the vanadium bonding environment and medium-range ordering of $\mathrm{V}(\mathrm{TCNE})_{\mathrm{x} \sim 2}$ films is essential to understanding their novel properties. Here we use STEM electron energy loss spectroscopy to characterize $\mathrm{V}(\mathrm{TCNE})_{\mathrm{x} \sim 2}$ films grown by chemical vapor deposition(CVD). We have established a sample transfer and preparation routine that allows us to make cross section lamella via focused ion beam milling with no oxidation detected by EELS analysis. With the use of a Gatan ${ }^{6}$ vacuum transfer holder, we have also been able to transfer and analyze plan view samples grown on various TEM grids. Our EELS data indicates that the valence state of the vanadium in the CVD grown films is $2+$ with no variation throughout the $\mathrm{V}(\mathrm{TCNE})_{\mathrm{x} \sim 2}$ layer. If the vanadium was in a perfect octahedral environment, we would expect to see splitting or asymmetry on the $\mathrm{L}_{2}$ peak. The lack of structure on the $\mathrm{L}_{2}$ peak in our EELS data (Figure1) indicates that the vanadium may be in a distorted octahedral environment. Figure 1 also shows the vanadium L edge of the same sample after oxidation. Interestingly, there is more structure on the vanadium $\mathrm{L}_{2,3}$ peaks in the oxidized spectrum which could signify a relaxed structure after oxidation. The corresponding $\mathrm{C}$ and $\mathrm{N}$ edges in Figure 2 also show a decrease in the $\mathrm{C}$ pi* peak of the oxidized sample leading us to believe that the oxygen is attacking the carbon-carbon double bond of the TCNE molecules.

In this contribution, we will further discuss using EELS to further analyze the vanadium valence state, bonding environment, stoichiometry, and band gap of V(TCNE)x films. We are also exploring the use of a pixelated detector (EMPAD) ${ }^{7}$ to probe the medium-range order. 


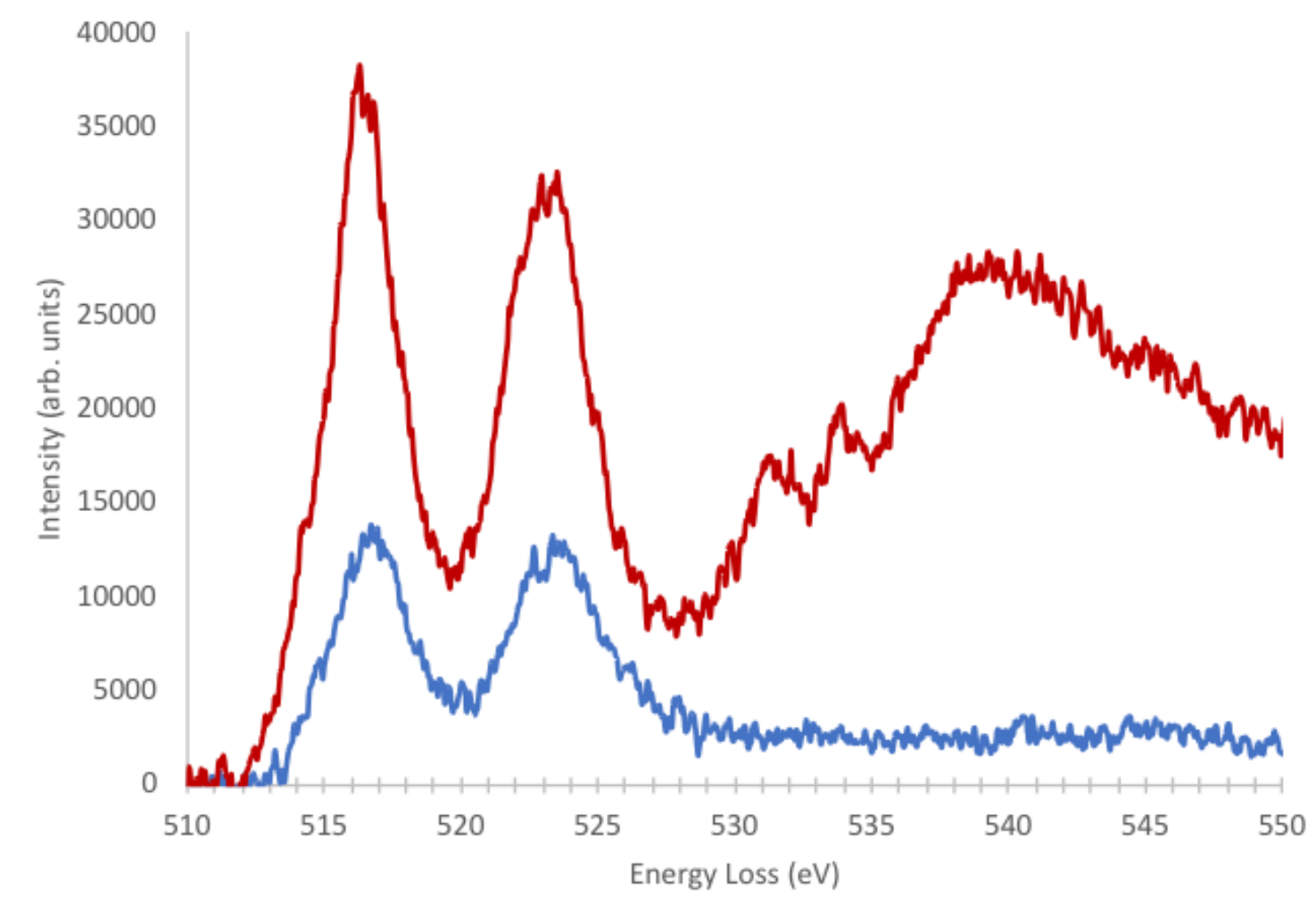

Figure 1. EELS spectra showing the V L2,3 peaks of the same sample before (blue) and after (red) oxidation.

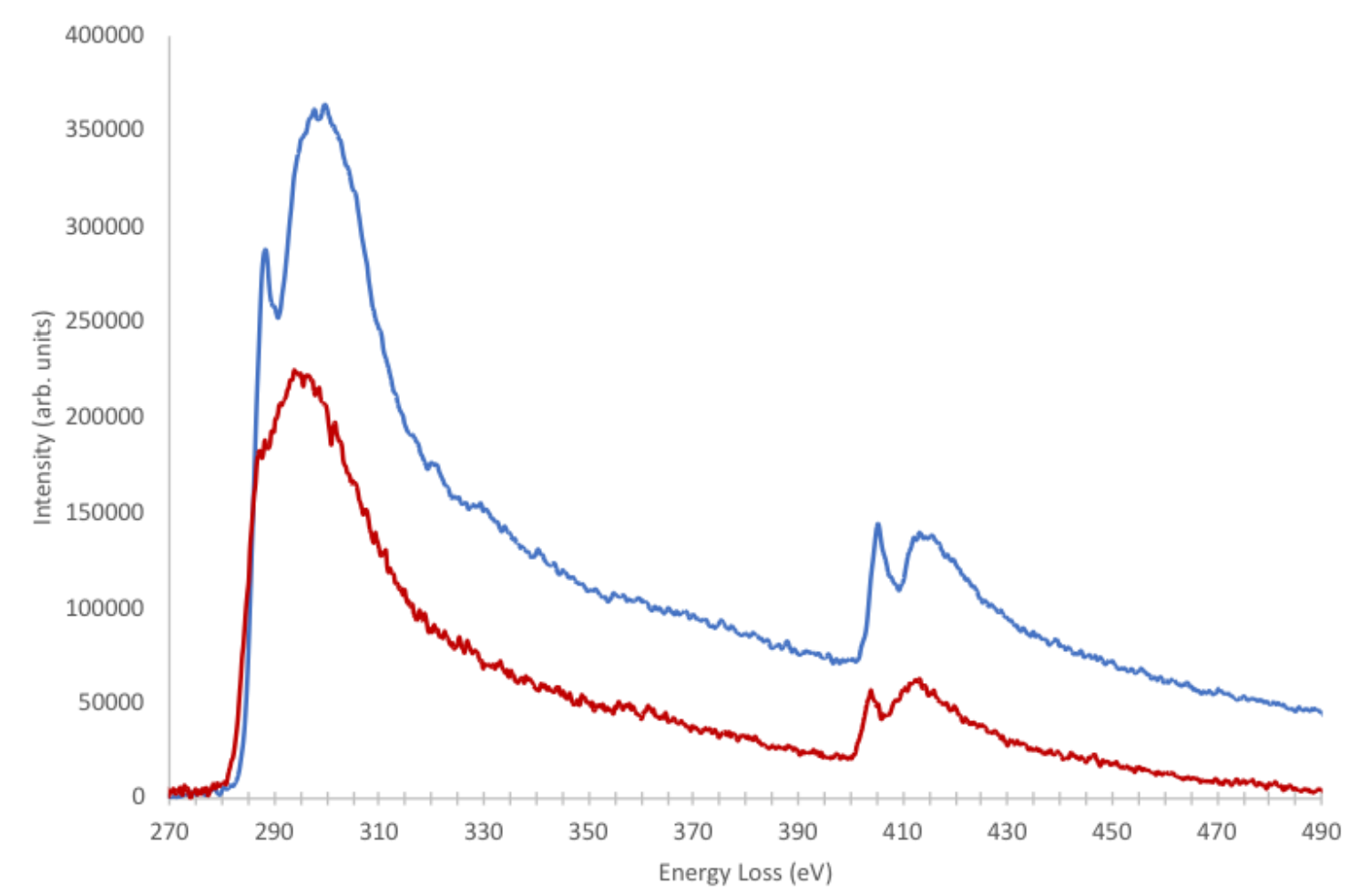

Figure 2. EELS spectra showing the $\mathrm{C}$ and $\mathrm{N}$ edges of the same sample before (blue) and after (red) oxidation. 


\section{References}

[1] Matsuura, H. et al. J. Phys. Soc. Jpn. 79, 034712 (2010).

[2] Haskel, D. et al. Physical Review B 70, 054422 (2004).

[3] Kortright, J. B. et al. Physical Review Letters 100, 257204 (2008).

[4] Lu, Y. et al. J. Mater. Chem. C. 3, 7363 (2015).

[5] Pokhodnya, K. I. et al. Advanced Materials 6, 12 (2000).

[6] www.gatan.com/products

[7] Tate, M. et al. Microscopy and Microanalysis 22, 237-249 (2016). 